\title{
SPG9A with the new occurrence of an ALDH18A1 mutation in a CMT1A family with PMP22 duplication: case report
}

\author{
Kishin Koh ${ }^{1} \mathbb{D}$, Ryusuke Takaki ${ }^{1,2}$, Hiroyuki Ishiura ${ }^{3} \mathbb{D}$, Shoji Tsuji, ${ }^{4,5}$ and Yoshihisa Takiyama ${ }^{1 *}$ (D)
}

\begin{abstract}
Background: ALDH18A1 mutations lead to delta-1-pyrroline-5-carboxylate-synthetase (P5CS) deficiency, which is a urea cycle-related disorder including SPG9A, SPG9B, autosomal dominant cutis laxa-3 (ADCL3), and autosomal recessive cutis laxa type $3 A(A R C L 3 A)$. These diseases exhibit a broad clinical spectrum, which makes the diagnosis of P5CS deficiency difficult. We report here a rare Japanese family including both patients with an ALDH18A1 mutation (SPG9A) and ones with CMT1A.

Case presentation: A Japanese family included five patients with the CMT phenotype and five with the HSP phenotype in four generations. The patients with the HSP phenotype showed a pure or complicated form, and intrafamilial clinical variability was noted. Genetically, FISH analysis revealed that two CMT patients had a PMP22 duplication (CMT1A). Exome analysis and Sanger sequencing revealed five HSP patients had an ALDH18A1 heterozygous mutation of c.755G > A, which led to SPG9A. Haplotype analysis revealed that the ALDH18A1 mutation must have newly occurred. To date, although de novo mutations of ALDH18A1 have been described in ADCL3A, they were not mentioned in SPG9A in earlier reports. Thus, this is the first SPG9A family with a de novo mutation or the new occurrence of gonadal mosaicism of ALDH18A1. Analysis of serum amino acid levels revealed that two SPG9A patients and two unaffected family members had low citrulline levels and one had a low level of ornithine.

Conclusions: Since the newly occurring ALDH18A1 mutation, c.755G > A, is the same as that in two ADHSP families and one sporadic patient with SPG9A reported previously, this genomic site might easily undergo mutation. The patients with the c.755G > A mutation in our family showed clinical variability of symptoms like in the earlier reported two families and one sporadic patient with this mutation. Further studies are required to clarify the relationship between the amino acid levels and clinical manifestations, which will reveal how P5CS deficiency influences disease phenotypes including ARCL3A, ADCL3, SPG9B, and SPG9A.
\end{abstract}

Keywords: SPG9A, ALDH18A1, de novo mutation, Gonadal mosaicism, Charcot-Marie-tooth disease, PMP22

\footnotetext{
* Correspondence: ytakiyama@yamanashi.ac.jp

'Department of Neurology, Graduate School of Medical Sciences, University of Yamanashi, Yamanashi 409-3898, Japan

Full list of author information is available at the end of the article
}

(c) The Author(s). 2021 Open Access This article is licensed under a Creative Commons Attribution 4.0 International License, which permits use, sharing, adaptation, distribution and reproduction in any medium or format, as long as you give appropriate credit to the original author(s) and the source, provide a link to the Creative Commons licence, and indicate if changes were made. The images or other third party material in this article are included in the article's Creative Commons licence, unless indicated otherwise in a credit line to the material. If material is not included in the article's Creative Commons licence and your intended use is not permitted by statutory regulation or exceeds the permitted use, you will need to obtain permission directly from the copyright holder. To view a copy of this licence, visit http://creativecommons.org/licenses/by/4.0/ The Creative Commons Public Domain Dedication waiver (http://creativecommons.org/publicdomain/zero/1.0/) applies to the data made available in this article, unless otherwise stated in a credit line to the data. 


\section{Background}

SPG9A and SPG9B are hereditary spastic paraplegias (HSPs) caused by dominant and recessive mutations of ALDH18A1, respectively. These diseases are classified as delta-1-pyrroline-5-carboxylate-synthedase (P5CS) deficiency [1], which is a urea cycle-related disorder. P5CS deficiency includes SPG9A [OMIM \#601162], SPG9B [OMIM \#616586], ADCL3 [OMIM \#616603], and ARCL3A [OMIM \#219150] [2]. The disease severity in P5CS deficiency might increase in the order of SPG9A < $\mathrm{SPG9B}<\mathrm{ADCL} 3 \leq \mathrm{ARCL3A}$ [2]. These diseases can be understood with the concept of a disease continuum caused by various levels of loss of P5CS function [2]. P5CS deficiency exhibits a broad clinical spectrum including cutis laxa, connective tissue weakness, global developmental delay, microcephaly, cataracts, vomiting, hypotonia, spasticity, pyramidal signs, motor disability, and corpus callosum hypotrophy with variable disease severity. Thus, it is difficult to diagnose P5CS deficiency only from clinical information.

We describe here a rare Japanese family with various neurological manifestations including neuropathy, pure HSP, and complicated HSP. Although these phenotypes were initially considered as a consequence of a disease, molecular genetic analysis revealed there are two diseases, SPG9A and Charcot-Marie-Tooth disease type 1A (CMT1A), in one family. In Japan, although SPG9B has been reported in two families so far $[3,4]$, this is the first family with SPG9A. Furthermore, haplotype analysis indicated SPG9A might have been caused by the new occurrence of an $A L D H 18 A 1$ mutation in this family.

\section{Case presentation}

\section{Patients and methods}

A pedigree chart is shown in Fig. 1. The clinical findings for each phenotype in representative patients are as follows.

Patient III-3 (68-year-old male) presented a phenotype of CMT. He showed gait disability when he was an elementary school student. In his 40s he exhibited clumsiness in his hands. These symptoms were slowly progressive. Neurological examination at age 68 revealed a steppage gait and his legs had the inverted champagne bottle appearance. The distal muscle power was moderately reduced. Tendon reflexes of the upper and lower limbs were decreased. Babinski signs were absent. A standardized electrophysiological study showed decreased conduction velocities of less than $38 \mathrm{~m} / \mathrm{s}$ in the median and tibial nerves.

Patient IV-1 (38-year-old male) presented a phenotype of a pure form of HSP. He showed a spastic gait when he was a high school student. Neurological examination at age 38 revealed a spastic gait, increased deep tendon reflexes of the lower limbs, and positive Babinski signs.

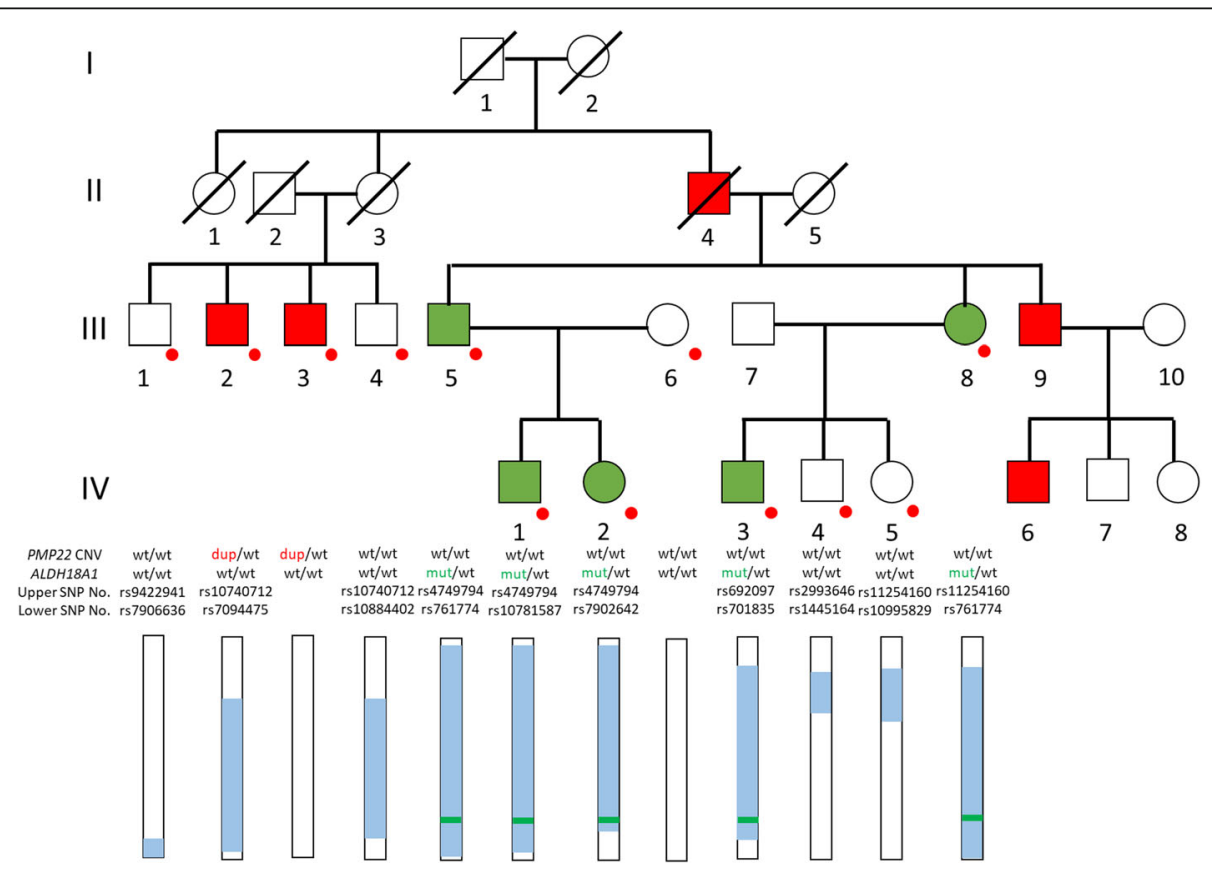

Fig. 1 Squares and circles indicate males and females, respectively. Red and green filling indicates a CMT phenotype and HSP phenotype, respectively. Red dots indicate participants from which DNA was taken. Rectangles with white or/and light blue shading indicate the haplotypes of chr17, which were the inferred haplotypes. Light blue indicates the same haplotype. The upper and lower limits of the SNP for each blue haplotype are denoted by "Upper or Lower SNP No." The green bars indicate the ALDH18A1 mutation and its position. wt: wild type, dup: PMP22 duplication, mut: ALDH18A1 mutation 
Table 1 Clinical features and serum amino acid levels of the patients in the present family and those in the earlier reported families

\begin{tabular}{|c|c|c|c|c|c|c|c|c|c|c|c|c|c|c|c|c|c|c|c|c|c|c|c|c|c|c|c|c|c|}
\hline \multirow[b]{2}{*}{ Patient } & \multicolumn{15}{|c|}{ Our family } & \multicolumn{9}{|c|}{ Ref. $[11,12]$} & \multicolumn{5}{|c|}{ Ref. [1] } \\
\hline & III-1 & III-4 & III-6 & IV.4 & IV-5 & $11-4$ & III-2 & III-3 & $1111-9$ & IV-6 & 111-5 & III-8 & IV-1 & IV-2 & IV-3 & $\mid-1$ & \begin{tabular}{|l|l|l|}
$\|-1$ & $\|$
\end{tabular} & $\|-4\|$ & \begin{tabular}{|c|c|}
$111-2$ & -1 \\
\end{tabular} & III-4 & IV-1 & $\mid$ IV -2 & IV-4 & IV-7 & $\# 5$ & H9 & $\# 11$ & $\# 12$ & $\# 25014$ \\
\hline Age & 73 & NA & 66 & 39 & 36 & 90 & 70 & 68 & NA & NA & 69 & 66 & 38 & 36 & 41 & NA & $60 N$ & NA $N$ & NA & NA & NA & NA & NA & NA & 52 & 50 & 48 & 20 & 76 \\
\hline Onset & - & - & - & - & - & NA & Child & Child & Child & $10 \mathrm{~s}$ & NA & $60 \mathrm{~s}$ & $10 \mathrm{~s}$ & NA & NA & NA & NA $N$ & NA & 5 & NA & 2 & 1 & NA & NA & 19 & 21 & 43 & 1 & 31 \\
\hline Sex & M & M & $\mathrm{F}$ & M & $\mathrm{F}$ & M & M & M & M & M & M & $\mathrm{F}$ & M & $\mathrm{F}$ & M & M & M & $\mathrm{F}$ & $\mathrm{F}$ & $\mathrm{F}$ & M & M & $\mathrm{F}$ & M & $\mathrm{F}$ & $\mathrm{F}$ & M & $\mathrm{F}$ & $\mathrm{F}$ \\
\hline $\begin{array}{l}\text { Cardinal } \\
\text { Phenotype }\end{array}$ & \multicolumn{5}{|c|}{ Unaffected family members } & \multicolumn{5}{|c|}{ CMT1A (PMP22 duplication) } & \multicolumn{19}{|c|}{ HSP(ALDH18A P.R252Q) } \\
\hline Ataxia & - & - & - & - & - & - & - & - & - & - & - & - & - & + & - & NA & - & + & - & NA & - & - & NA & NA & - & - & - & - & - \\
\hline $\begin{array}{c}\text { Mental } \\
\text { Retardation }\end{array}$ & - & NA & - & - & - & - & - & - & NA & + & - & - & + & + & - & NA & - & $\mathrm{NA}$ & . & NA & + & . & $\mathrm{NA}$ & NA & . & - & - & - & $\cdot$ \\
\hline Tremor & - & NA & - & - & - & + & - & - & NA & NA & - & - & - & + & - & NA & - & NA & - & NA & - & - & NA & NA & - & - & - & - & - \\
\hline Tendon reflex & $\downarrow$ & NA & $\rightarrow$ & $\rightarrow$ & $\rightarrow$ & NA & $\rightarrow$ & $\downarrow$ & NA & NA & $\uparrow$ & $\uparrow$ & $\uparrow$ & $\uparrow$ & $\rightarrow$ & NA & $\uparrow$ & $\uparrow$ & $\uparrow$ & NA & $\uparrow$ & $\rightarrow$ & NA & NA & $\uparrow$ & $\uparrow$ & $\uparrow$ & $\uparrow$ & $\uparrow$ \\
\hline Babinski & $\%$ & NA & $\%$ & $\%$ & $\%$ & NA & $\%$ & $\%$ & NA & NA & $\%$ & $\%$ & $+/+$ & $+/+$ & $\%$ & NA & $\mathrm{NA}+$ & $+1++$ & $+/+$ & NA & NA & $\%$ & NA & NA & $+/+$ & $+1+$ & $+/+$ & $+/+$ & $+/+$ \\
\hline Chaddock & $\%$ & NA & $\%$ & $\%$ & $\%$ & NA & $\%$ & $\%$ & NA & NA & $\%$ & $\%$ & t/t & $\%$ & $\%$ & NA & NA & NA & NA & NA & NA & NA & NA & NA & NA & NA & NA & NA & NA \\
\hline Gait & normal & normal & normal & normal & normal & NA & steppage & normal & steppage & NA & normal & spastic & spastic & $\begin{array}{c}\text { Spastic } \\
\text { Steppage }\end{array}$ & normal & NA & NA & NA & NA & $N A$ & $\mathrm{NA}$ & NA & $\mathrm{NA}$ & NA & Spastic & Spastic & Spastic & Spastic & Spastic \\
\hline Amyotrophy & - & NA & - & - & - & + & + & + & + & NA & - & - & + & + & - & NA & NA $N$ & NA & + & NA & NA & - & NA & NA & NA & NA & NA & NA & NA \\
\hline Ornithine & NA & NA & NA & 77.3 & 76.2 & NA & NA & NA & NA & NA & NA & 84.6 & NA & $30 \downarrow$ & 78.9 & NA & NA $N$ & NA & NA & NA & $\mathrm{NA}$ & NA & NA & NA & NA & NA & NA & NA & NA \\
\hline Citrulline & NA & NA & NA & $14.6 \downarrow$ & $9.3 \downarrow$ & NA & NA & NA & NA & NA & NA & $13.2 \downarrow$ & NA & $7.8 \downarrow$ & $12.5 \downarrow$ & NA & NA & NA & NA & NA & NA & NA & $\mathrm{NA}$ & NA & NA & NA & NA & NA & NA \\
\hline Arginine & NA & NA & NA & 53.9 & $36.6 \downarrow$ & NA & NA & NA & NA & NA & NA & 48.7 & NA & 64.3 & 50.4 & NA & NA & NA & NA & NA & NA & NA & NA & NA & NA & NA & NA & NA & NA \\
\hline Isoleucine & NA & NA & NA & 77.2 & 66.1 & NA & NA & NA & NA & NA & NA & 83.8 & NA & 42 & $87.9 \uparrow$ & NA & NA & NA & NA & NA & NA & NA & NA & NA & NA & NA & NA & NA & NA \\
\hline Glutamine & NA & NA & NA & 587.1 & 605.3 & NA & NA & NA & NA & NA & NA & 625.1 & NA & 690.8 & $783.3 \uparrow$ & NA & NA & NA & NA & NA & NA & NA & NA & NA & NA & NA & NA & NA & NA \\
\hline Alanine & NA & NA & NA & 465.9 & 456.5 & NA & NA & NA & NA & NA & NA & 406.8 & NA & 309.7 & $596.1 \uparrow$ & NA & NA & NA & NA & NA & NA & NA & NA & NA & NA & NA & NA & NA & NA \\
\hline oxyproi & NA & NA & NA & $9.9 \uparrow$ & 10.1 & NA & NA & NA & NA & NA & NA & 7.6 & NA & 16 & $19.7 \uparrow$ & NA & NA & NA & NA & NA & NA & NA & NA & NA & NA & NA & NA & NA & \\
\hline
\end{tabular}

HSP: hereditary spastic paraplegia, CMT: Charcot-Marie-Tooth disease, NA: not available, F: female, M: male, $\rightarrow$ : normal, $\uparrow:$ increased, $\downarrow:$ decreased

HSP hereditary spastic paraplegia, CMT Charcot-Marie-Tooth disease, NA not available, $F$ female, $M$ male, $\rightarrow:$ normal, $\uparrow:$ increased, $\downarrow:$ decreased

Patient IV-2 (36-year-old female) presented a phenotype of a complicated form of HSP. She showed gait disability when she was an elementary school student. In her 30s she exhibited dysarthria and dysphagia. Neurological examination at age 36 revealed a spastic and ataxic gait, and positive Babinski signs. She did not show any muscle weakness.

In summary, Patients II-4, III-2, III-3, III-9, and IV-6 had a phenotype of CMT, Patients III-5, III-8, IV-1, and IV-3 a phenotype of a pure form of HSP, and Patient IV-2 a phenotype of a complicated form of HSP (Table 1).

We screened for PMP22 duplications/deletions, which cause CMT1A, by fluorescence in situ hybridization (FISH) at first because we clinically diagnosed Patient III-3 as having a CMT1 phenotype. After screening for CMT1A, we performed target-sequencing for known HSP and CMT causative genes (Table 2) in Patients III3 and IV-2. We filtered variations by using dbSNP146 [5], 1000 Genomes [6], HGVD [7], and iJGVD [8]. We evaluated the functional prediction of $A L D H 18 A 1$ mutations by means of in silico algorithms using the Combined Annotation Dependent Depletion (CADD) score

Table 2 HSP and CMT genes checked

(ATL1, SPAST, NIPA1, KIAA0196, ALDH18A1, KIF5A, RTN2, HSPD1, BSCL2, ATSV, REEP1, ZFYVE27, SLC33A1, REEP2, CPT1C, CYP7B1, SPG7, SPG11, ZFYVE26, ERLIN2, SPG20, ACP33, B4GALNT1, DDHD1, FA2H, PNPLA6, C9orf12, GJA2, AP4B1, KIAA0415, TECPR2, AP4M1, AP4E1, AP4S1, VPS37A, DDHD2, c120rf65, CYP2U1, TFG, KIF1C, USP8, WDR48, ARL6IP1, ERLIN1, AMPD2, ENTPD1, ARSI, PGAP1, FLRT1, RAB3GAP2, MARS, ZFR, IBA57, MAG, MPZ, LITAF, EGR2, NEFL, FBLN5, KARS, SOX10, GJB3, ARHGEF10, G GNB4, HARS, GDAP, MTMR2, SBF2, SBF1, SH3TC2, NDRG1, EGR2, PRX, HK1, FGD4, FIG 4, SURF1, CTDP1, ASAH1, PMM2, GALC, ARSA, PHYH, PEX7, ABHD12, DNAJC3, GJB1, MFN2, KIF1B, RAB7, TRPV4, GARS, HSPB1, GDAP1, HSPB8, DNM2, AARS, DYNC1H1, LRSAM1, DHT, DNAJB2, MARS, NAGLU, HARS, VCP, MORC2, LMNA, MED25, HSPB1, DNM2, YARS, INF2, GNB4, GDAP)
[9]. We applied Sanger sequencing to perform a cosegregation study in this family. Furthermore, we performed haplotype analysis by using SNP typing with Affymetrix Genome-Wide Human SNP array 6.0. Haplotypes were reconstructed with Allegro v2 [10]. In addition, we determined the serum amino acid levels in Patients III-8, IV-2, 3, 4, and 5.

\section{Results}

We identified duplication of PMP22 in Patients III-2 and III-3, who presented a CMT phenotype on FISH analysis. On whole exome analysis in Patient IV-2, we identified two candidate variations in GARS (c.374A $>\mathrm{T}$, p.E125V) and ALDH18A1 (c.755G > A, p.R252Q) in Patient IV-2. The variation in GARS did not co-segregate with CMT patients in this family. On the other hand, the ALDH18A1 variation co-segregated with patients with a phenotype of HSP (Patients III-5, 8, IV-1, IV-2, and IV-3). This variation was not detected in dbSNP146 [5], 1000 genome project [6], HGVD [7], or iJGVD [8], and the CADD score was 26.8 (deleterious $>20$ ). Moreover, this variation was previously reported as a diseasecausing variant in SPG9A [1, 11-13]. These results showed Patients III-2 and III-3 had CMT1A, and Patients III-5, III-8, IV-1, IV-2, and IV-3 had SPG9A. Furthermore, haplotype analysis revealed that individuals I1, II-3, II-4, III-2, III-4, III-5, III-8, and IV-1-3 had the same haplotype including the ALDH18A1 mutation position (Fig. 1). However, the ALDH18A1 mutation was only identified in individuals III-5, III- 8 , IV-1, IV-2, and IV-3. This indicated that the ALDH18A1 mutation may have occurred as a de novo mutation on transmission to individual II-4 from either the parents or the new occurrence of gonadal mosaicism. 
Analysis of serum amino acid levels revealed that all participants including three SPG9A patients and two unaffected family members had low levels of citrulline and Patient IV-2 had low levels of citrulline and ornithine.

\section{Discussion and conclusions}

This study revealed SPG9A patients and CMT1A ones in one family. Haplotype analysis revealed that an ALDH18A1 mutation might have newly occurred as a de novo mutation from the 1st generation to Patient II-4 or the new occurrence of gonadal mosaicism in Patient II4. To date, although de novo mutations of ALDH18A1 have been described in ADCL3A [14-16], they were not reported in SPG9A in earlier reports $[1,11,17]$. Thus, this is the first SPG9A family with a de novo mutation or the new occurrence of gonadal mosaicism of $A L D H 18 A 1$. It was transmitted to five individuals with a HSP phenotype.

Since the newly occurring mutation of ALDH18A1, c.755G > A, has been reported in two families and one sporadic case so far $[1,11,13]$, this genetic site might be important because it was shown that the c.755G > A mutation promotes the dissociation of a hexamer into dimers [11]. This increased tendency of the c.755G $>$ A mutant form of P5CS to dissociate must reflect disturbance by the mutation of the intersubunit interactions within the hexamer. According to in silico analysis and the previous report, this mutation is disease-causing. SPG9A was reported to have both pure and complicated forms $[1,4]$. It was also reported that the same ALDH18A1 mutation in the same family caused variability of symptoms [1]. The present family also had pure and complicated forms of HSP. The previously reported two families and one sporadic case also exhibited variability of symptoms, i.e., pure or complicated forms, severe or mild. Therefore, it seems to be difficult to determine the genotype-phenotype correlation in SPG9A. However, the ALDH18A1 mutation would exhibit a genotype-phenotype correlation because ALDH18A1 deficiency shows several phenotypes, i.e., SPG9A, SPG9B, ADCL3, and ARCL3, which reflect the P5CS function. Fluctuation of the serum amino acid levels was reported in ADCL3 [16]. It might be considered that fluctuation of the serum amino acid levels would reflect severity and mutation would reflect P5CS deficiency.

Our data show that all participants with the ALDH18A1 mutation had low citrulline levels, although individuals without the mutation also showed low citrulline levels. On the other hand, only one patient with a low ornithine level had a complicated form of HSP. However, one report on ALDH18A1 mutations showed instability of the serum amino acid levels [7]. Therefore, further studies are required to clarify the relationship between the amino acid levels and clinical manifestations. This will reveal how P5CS deficiency influences disease phenotypes including ARCL3A, ADCL, SPG9B, and SPG9A.

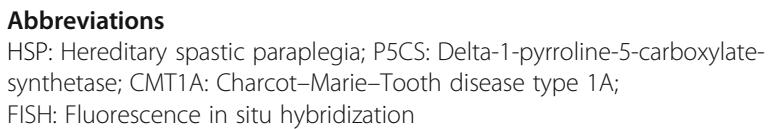

\section{Acknowledgements}

We thank the patients for participating in the present study. We also thank Dr. Shuji Hanyu, Department of Neurology, lida Hospital, for his support in the clinical examination of the patients.

\section{Authors' contributions}

YT designed and organized the study. KK, RT and $Y T$ acquired the clinical and laboratory data. KK, HI, ST and YT carried out the molecular genetic studies, and analyzed the molecular and clinical data. KK wrote the manuscript, which was edited by HI, ST and YT. All authors have read and approved the final version of the manuscript submitted by $Y T$.

\section{Funding}

The following programs provided financial support to the conduct of this research, including the study design, collection, analysis, and interpretation of data, and writing of the manuscript: Grants-in-Aid from the Research Committee for Ataxic Disease (Y.T.), the Ministry of Health, Labor and Welfare, Japan, and JSPS KAKENHI Grant Numbers 17 K17772 (K. K.), 19 K16910 (K. K.), and JP18K07495 (Y. T.) from the Ministry of Education, Culture, Sports, Science, and Technology, Japan.

\section{Availability of data and materials}

The data used and/or analyzed during the current study are available from the corresponding author on reasonable request.

\section{Ethics approval and consent to participate}

This study was approved by the Research Ethics Committee of Faculty of Medicine, University of Yamanashi (\#734 and \#953), and written informed consent for participation was obtained from all the participants.

\section{Consent for publication}

Written informed consent for publication was obtained from all the participants.

\section{Competing interests}

The authors disclose no potential conflicts of interest.

\section{Author details}

${ }^{1}$ Department of Neurology, Graduate School of Medical Sciences, University of Yamanashi, Yamanashi 409-3898, Japan. ${ }^{2}$ Department of Neurology, lida Hospital, Nagano 395-8505, Japan. ${ }^{3}$ Department of Neurology, Graduate School of Medicine, The University of Tokyo, Tokyo 113-8655, Japan. ${ }^{4}$ Department of Molecular Neurology, University of Tokyo, Graduate School of Medicine, Tokyo 113-8655, Japan. ${ }^{5}$ Department of Neurology, International University of Health and Welfare, Chiba 286-8686, Japan.

Received: 20 November 2020 Accepted: 2 February 2021

Published online: 11 February 2021

\section{References}

1. Coutelier M, Goizet C, Durr A, Habarou F, Morais S, Dionne-Laporte A, Tao F, Konop J, Stoll M, Charles P, Jacoupy M, Matusiak R, Alonso I, Tallaksen C, Mairey M, Kennerson M, Gaussen M, Schule R, Janin M, Morice-Picard F, Durand CM, Depienne C, Calvas P, Coutinho P, Saudubray JM, Rouleau G, Brice A, Nicholson G, Dariou F, Loureiro JL, Zuchner S, Ottolenghi C, Mochel F, Stevanin G. Alteration of ornithine metabolism leads to dominant and recessive hereditary spastic paraplegia. Brain. 2015;138:2191-205.

2. Marco-Marín C, Escamilla-Honrubia JM, Llácer JL, Seri M, Panza E, Rubio V. $\Delta$ 1-Pyrroline-5-carboxylate synthetase deficiency: an emergent multifaceted urea cycle-related disorder. J Inherit Metab Dis. 2020;43:657-70. 
3. Koh K, Ishiura H, Tsuji S, Takiyama Y. JASPAC: Japan spastic paraplegia research consortium. Brain Sci. 2018;8:153.

4. Koh K, Ishiura H, Beppu M, Shimazaki H, Ichinose Y, Mitsui J, Kuwabara S, Tsuji S, Takiyama Y, Japan Spastic Paraplegia Research Consortium. Novel mutations in the ALDH18A1 gene in complicated hereditary spastic paraplegia with cerebellar ataxia and cognitive impairment. J Hum Genet. 2018;63:1009-13.

5. Sherry ST, Ward MH, Kholodov M, Baker J, Phan L, Smigielski EM, Sirotkin K. dbSNP: the NCBI database of genetic variation. Nucleic Acids Res. 2001;29: 308-11.

6. The 1000 Genomes Project Consortium. A global reference for human genetic variation. Nature 2015;526:68-74.

7. Higasa K, Miyake N, Yoshimura J, Okamura K, Niihori T, Saitsu H, Doi K, Shimizu M, Nakabayashi K, Aoki Y, Tsurusaki Y, Morishita S, Kawaguchi T, Migita O, Nakayama K, Nakashima M, Mitsui J, Narahara M, Hayashi K, Funayama R, Yamaguchi D, Ishiura H, Ko W, Hata K, Nagashima T, Yamada R, Matsubara Y, Umezawa A, Tsuji S, Matsumoto N, Matsuda F. Human genetic variation database, a reference database of genetic variations in the Japanese population. J Hum Genet. 2016;61:547-53.

8. Tadaka S, Saigusa D, Motoike IN, Inoue J, Aoki Y, Shirota M, Koshiba S, Yamamoto M, Kinoshida K. jMorp: Japanese multi omics reference panel. Nucleic Acids res. 2018:46:D551-7.

9. Rentzsch P, Witten D, Cooper GM, Shendure J, Kircher M. CADD: predicting the deleteriousness of variants throughout the human genome. Nucleic Acids Res. 2019:47:D886-94.

10. Guadjartsson DF, Thorvaldsson T, Kong A, Gunnarsson G, Ingolfsdottir A. Allegro version 2. Nat Genet. 2005;37:1015-6.

11. Panza E, Escamilla-Honrubia JM, Marco-Marín C, Gougeard N, Michele GD, Morra VB, Liguori R, Salviati L, Donati MA, Cusano R, Pippucci T, Ravazzolo R, Németh AH, Smithson S, Davies S, Hurst JA, Bordo D, Rubio V, Seri M. ALDH18A1 gene mutations cause dominant spastic paraplegia SPG9: loss of function effect and plausibility of a dominant negative mechanism. Brain. 2016;139:e3

12. Slavotinek AM, Pike M, Mills K K Hurst JA. Cataracts, motor system disorder, short stature, learning difficulties, and skeletal abnormalities: a new syndrome? Am J Med Genet. 1996;62:42-7.

13. Ngo KJ, Rexach JE, Lee H, Petty LE, Perlman S, Valera JM, Deignan JL, Mao Y, Aker M, Posey JE, Jhangiani SN, Coban-Akdemir ZH, Boerwinkle E, Donna M, Nelson AB, Hassin-Baer S, Poke G, Neas K, Geschwind MD, Grody WW, Gibbs R, Geschwind DH, Lupski JR, Below JE, Nelson SF, Fogel BL. A diagnostic ceiling for exome sequencing in cerebellar ataxia and related neurological disorders. Hum Mutat. 2020;41:487-501.

14. Yoshimura A, Yuan JH, Hashiguchi A, Ando M, Higuchi Y, Nakamura T, Okamoto Y, Nakagawa M, Takashima H. Genetic profile and onset features of 1005 patients with Charcot-Marie-tooth disease in Japan. J Neurol Neurosurg Psychiatry. 2019;90:195-202.

15. Fischer-Zirnsak B, Escande-Beillard N, Ganesh J, Tan YX, Al-Bughaili M, Lin AE, Sahai I, Bahena P, Reichert SL, Loh A, Wright GD, Liu J, Rahikkala E, Pivnick EK, Choudhri AF, Kruger U, Zemojtel T, van Ravenswaaij-Arts C, Mostafavi R, Stolte-Dijkstra I, Symoens S, Pajunen L, Al-Gazali L, Meierhofer D, Robinson SP, Mundlos S, Villarroel CE, Byers P, Masri A, Robertson SP, Schwarze U, Callewaert B, Reversade B, Kornak U. Recurrent de novo mutations affecting residue Arg138 of pyrroline-5-carboxylate synthase cause a progeroid form of autosomal-dominant cutis laxa. Am J Hum Genet. 2015;97:483-92.

16. Nozaki F, Kusunoki T, Okamoto N, Yamamoto Y, Miya F, Tsunoda T, Kosaki K, Kumada T, Shibata M, Fujii T. ALDH18A-related cutis laxa syndrome with cyclic vomiting. Brain Dev. 2016;38:678-84.

17. Marelli C, Badiou S, Genestet S, Larrieu L, Damier P, Camu W, Planes M, Koenig M, Guissart C. Autosomal dominant SPG9: intrafamilial variability and onset during pregnancy. Neurol Sci. 2020;41:1931-3.

\section{Publisher's Note}

Springer Nature remains neutral with regard to jurisdictional claims in published maps and institutional affiliations.

\section{Ready to submit your research? Choose BMC and benefit from:}

- fast, convenient online submission

- thorough peer review by experienced researchers in your field

- rapid publication on acceptance

- support for research data, including large and complex data types

- gold Open Access which fosters wider collaboration and increased citations

- maximum visibility for your research: over $100 \mathrm{M}$ website views per year

At BMC, research is always in progress.

Learn more biomedcentral.com/submissions 